\title{
The Role of uPA and uPA Inhibitors in Breast Cancer
}

\author{
Paul Bevan Carola Mala
}

Wilex AG, Munich, Germany

In order for primary tumours to metastasise, tumour cells must undergo a complex series of steps, including detachment from the primary tumour, degradation of basement membranes, cell migration through the extracellular matrix (ECM), intravasation into the circulation, attachment and extravasation at a distant organ site, and cell division and growth of a new tumour colony. For all these steps, the urokinase plasminogen activator system (uPAS), along with members of the matrix metalloproteases (MMPs), has long been known to play a key role.

The urokinase-type plasminogen activator (uPA), when bound to its cellular receptor, uPAR, efficiently converts plasminogen into the serine protease plasmin, which then facilitates the release of several other proteolytic enzymes including gelatinase, fibronectin, fibrin, laminin, and latent forms of collagenase needed to degrade physical barriers to cell movement.

Binding of uPA to its receptor also triggers other important biological effects, including chemotaxis, migration, invasion, adhesion and proliferation. The binding also exposes the vitronectin-interacting surface of the receptor and modulates cell adhesion through the interaction with receptors of the integrin family, causing induction of actin cytoskeleton rearrangement and cell spreading. uPA binding to its receptor also plays an important role in protecting cells from apoptosis due to cell detachment (fig. 1).

uPA itself is activated through plasmin-mediated cleavage after binding to its receptor. Increased levels of active receptor-bound uPA lead to increased co-localised plasminogen binding on the cell surface, initiating a positive feedback mechanism leading to amplification of plasmin activity at the fronts of migrating cells.

Plasminogen activator inhibitor-1 (PAI-1) and the plasmin inhibitor $\alpha 2$-antiplasmin are considered the most important inhibitors of the uPAS. PAI-1 binds to active uPA in complex with uPAR and brings about the internalisation of the whole uPAR-uPA-PAI-1 complex. uPA-PAI-1 is degraded in lysosomes while the UPAR is recycled back to the cell surface.

Given the central role of the system in metastasis, it is not surprising that researchers have reported the clinical relevance of the UPAS in a variety of solid tumours, including breast, ovarian, oesophageal, gastric, pancreatic, colorectal, lung and liver cancer. Elevated levels of uPA and/or PAI-1 in tumour tissue extracts are associated with increased tumour aggressiveness and poor patient outcome. Particularly in breast cancer, where several retrospective and one large prospective trial proved the clinical relevance at the highest level of evidence (LOE-1), the determination of tissue levels of uPA are recommended for decision-making and clinical management of the patients in the ASCO guidelines (American Society of Clinical Oncology 2007 Up-

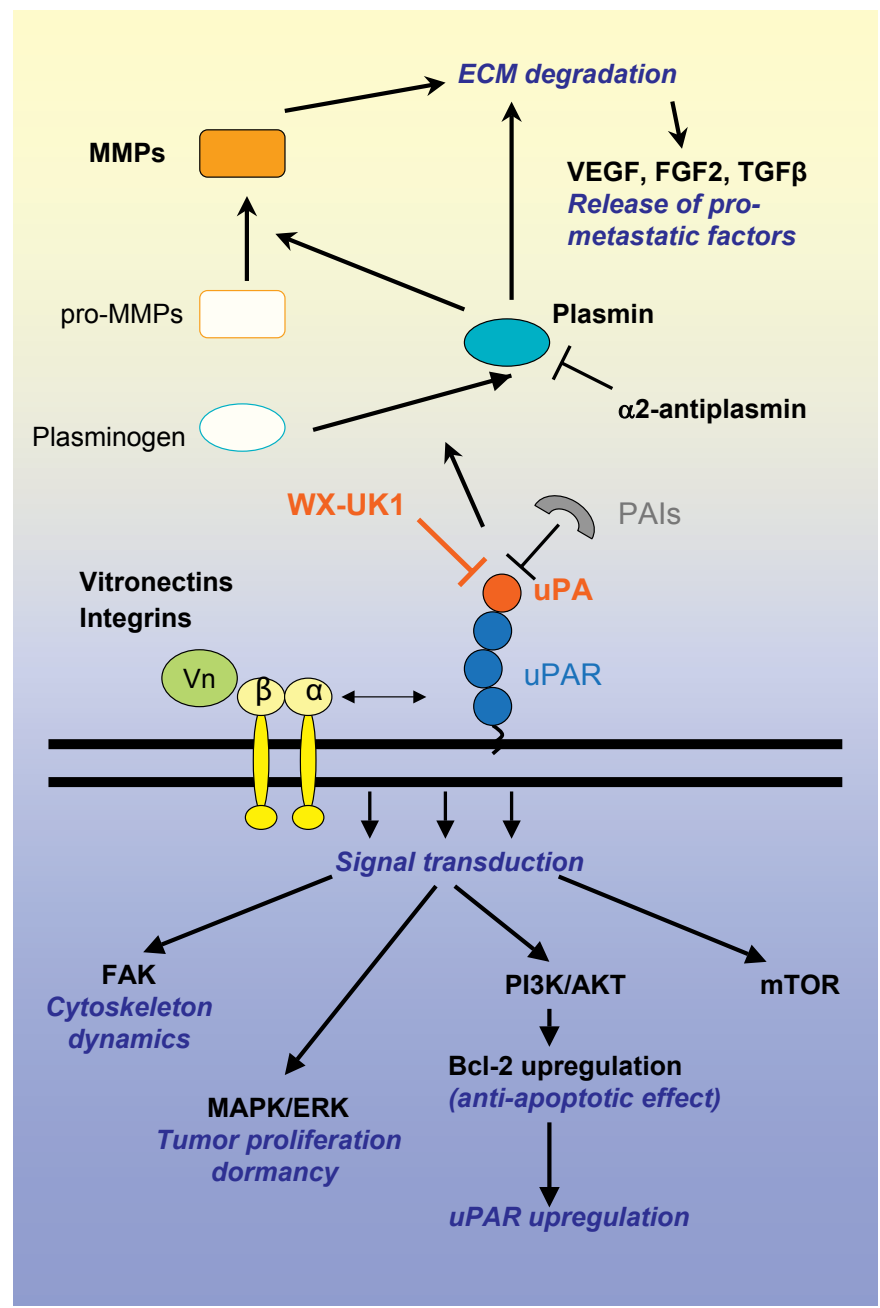

Fig. 1. The urokinase plasminogen activator system: components and biological functions. uPA, Urokinase-type plasminogen activator; uPAR, urokinase-type plasminogen activator receptor; PAI, plasminogen activator inhibitor; Bcl-2, B cell lymphoma 2; ECM, extracellular matrix; ERK, extracellular signal-regulated kinase; FAK, focal adhesion kinase; FGF-2, fibroblast growth factor 2; MAPK, mitogen-activated protein kinase; MMPs, matrix metalloproteinases; mTOR, mammalian target of rapamycin; PI3K, phosphatidylinositol 3-kinase; TGF- $\beta$, transforming growth factor beta; VEGF, vascular endothelial growth factor; Vn, vitronectin.

\section{KARGER}

Fax +497614520714

Information@Karger.de

www.karger.com
(C) 2008 S. Karger GmbH, Freiburg

Accessible online at:

www.karger.com/brc 
date of Recommendations for the Use of Tumour Markers in Breast Cancer). Indeed, uPA/PAI-1 is the only biomarker to have been conferred with LOE-1 as a definitive prognostic marker of poor disease outcome in early breast cancer. Furthermore, the ASCO guideline also considers the components of the uPAS to be promising targets for future therapeutic studies. Enthusiasm for this approach is nourished by the fact that the inhibition of the uPAS lacks toxicity, as demonstrated in UPA- or uPAR-deficient mice.

Another marker, HER2, has also been shown to be associated with a poor prognosis in breast cancer patients. The gene, the HER2neu proto-oncogene, is amplified in $25 \%$ of invasive breast cancers, resulting in a decreased time of disease-free survival and other markers of poor prognosis. Interestingly, there seems to be an excellent correlation between both HER2 and uPAR overexpression and their re- spective gene amplifications. Furthermore, in patients with HER2overexpressing tumours, co-amplification of HER2 and UPAR in the same tumour cell has been demonstrated. This would seem to support the hypothesis that combination treatment of breast cancer patients with inhibitors of HER 2 and uPA may have advantages compared to treatment with either agent alone.

The first inhibitors of uPA have now been tested in oncology trials worldwide, and one of the compounds, WX-671, is currently being evaluated in two international trials in combination with chemotherapy.

This supplement brings together a collection of manuscripts from plenary papers by leading researchers, delivered at the satellite symposium entitled 'uPA Inhibitors - First Clinical Experience in Solid Tumours' held in Berlin in April 2008 during the European Breast Cancer Conference (EBCC6). 